\title{
MULTIPLIERS ON COMPACT GROUPS
}

\author{
IAN INGLIS ${ }^{1}$
}

AbSTRACT. We give some sufficient conditions for a function on a compact totally disconnected abelian group to be an $L^{p}$ Fourier multiplier.

1. Introduction. Let $X$ denote a compact abelian group with a strictly decreasing sequence of open compact subgroups $\left\{X_{n}\right\}_{0}^{\infty}$ such that $\cup X_{n}=X$ $\cap X_{n}=\{0\}$ and $2 \leqslant\left|X_{n}\right| \cdot\left|X_{n+1}\right|^{-1} \leqslant b$, where $|S|$ denotes the Haar measure of a set $S$. Let $G$ denote the dual of $X$ and $G_{n}$ the annihilator of $X_{n}$ in $G$; thus $\left\{G_{n}\right\}$ is an increasing sequence of open compact subgroups of $G, \cup G_{n}=G$, $\cap G_{n}=\{0\}$ and $2 \leqslant\left|G_{n+1}\right| \cdot\left|G_{n}\right|^{-1} \leqslant b$. We denote by $d \chi$ and $d x$ the Haar measures on $X$ and $G$ respectively, and assume that these are adjusted so that the inversion theorem holds.

If $\phi \in L^{\infty}(X)$ then $\phi$ defines a bounded linear operator $T_{\phi}$ on $L^{2}(G)$ via the formula

$$
\left(T_{\phi} f\right)^{\wedge}=\phi \hat{f} .
$$

We say that $\phi$ is an $L^{2}$ Fourier multiplier. Similarly for $1 \leqslant p \leqslant \infty$, we say that $\phi$ is an $L^{p}$ Fourier multiplier, (and write $\phi \in M_{p}(X)$ ), if there exists a number $B$ such that

$$
\left\|T_{\phi} f\right\|_{p} \leqslant B\|f\|_{p}
$$

for all $f$ in $L^{p} \cap L^{2}(G)$; we write $\|\phi\|_{M_{p}}$ for the smallest value of $B$ for which (1) holds. It is well known that $M_{p}=M_{p^{\prime}}\left(\right.$ where, as always $(1 / p)+\left(1 / p^{\prime}\right)=$ 1) and

$$
A(X)=M_{1}(X) \subseteq M_{p}(X) \subseteq M_{q}(X) \subseteq M_{2}(X)=L^{\infty}(X)
$$

when $1 \leqslant p \leqslant q \leqslant 2$ and where $A(X)$ is the space of Fourier transforms of integrable functions with the inherited norm. A wealth of information about multipliers is contained in the book [1] of Edwards and Gaudry.

2. Suppose $1<\theta<\infty$; for $n \geqslant 0$ we define the subgroup $X_{n}^{\theta}$ of $X$ by the formula

$$
X_{n}^{\theta}=X_{j} \quad \text { where }\left|X_{j}\right| \geqslant\left|X_{n}\right|^{\theta}>\left|X_{j+1}\right| .
$$

Our main result is the following:

Received by the editors June 1, 1977.

AMS (MOS) subject classifications (1970). Primary 42A18; Secondary 42A40, 43A70.

Key words and phrases. Compact abelian group, Fourier multiplier.

${ }^{1}$ The author was supported by the National Research Council of Italy.

C American Mathematical Society 1978 
THEOREM. Suppose $\phi$ is a function on $X$ constant on cosets of $X_{n}^{\theta}$ outside $X_{n}$. If

$$
|\phi(\chi)| \leqslant B\left|X_{n}\right|^{(\theta-1) / 2} \text { when } \chi \in X_{n-1} \backslash X_{n}
$$

for some constant $B$ independent of $n$, then $\phi \in M_{p}(X)$ for $1<p<\infty$.

The proof employed is singular-integral in spirit, although no use is made of Calderón-Zygmund type covering lemmas. We need the following:

LEMMA. For $1<\theta<\infty$ we define the subgroup $G_{n}^{\theta}$ of $G$ by the formula

$$
G_{n}^{\theta}=G_{j} \text { where }\left|G_{j}\right|<\left|G_{n}\right|^{\theta}<\left|G_{j+1}\right| \text {. }
$$

In other words, $G_{n}^{\theta}$ is the annihilator of $X_{n}^{\theta}$. Suppose $k$ is an integrable function on $G$, constant on cosets of $G_{n}$ outside $G_{n}^{\theta}$. If

$$
|\hat{k}(\chi)|<B\left|X_{n}\right|^{(\theta-1) / 2+\beta} \text { when } \chi \in X_{n-1} \backslash X_{n}
$$

for some $\beta>0$, then

$$
\|k * f\|_{\infty} \leqslant B \cdot C \cdot\|f\|_{\infty}
$$

where $C$ is a constant independent of $\|k\|_{1}$.

Proof. Fix $f$ in $L^{\infty}$. By translation invariance it suffices to show that, for every $N \geqslant 0$,

$$
\left.|| G_{N}\right|^{-1} \int_{G_{N}} k * f d x \mid \leqslant B \cdot C\|f\|_{\infty} .
$$

Fix $N$ and write $f=f_{1}+f_{2}$ where $f_{1}=f \cdot \xi_{G_{N+1}^{\theta}}\left(\xi_{S}\right.$ denotes the indicator function of the set $S$ ). Then

$$
\begin{aligned}
\left.|| G_{N}\right|^{-1} \int_{G_{N}} k * f_{1} d x \mid & =\left|D_{N} * k * f_{1}(0)\right| \text { where } D_{n}=\xi_{G_{n}} \cdot\left|G_{n}\right|^{-1} \\
& =\left|\int_{X_{N}} \hat{k} \hat{f}_{1} d x\right| \\
& <B\left|X_{N}\right|^{(\theta-1) / 2+\beta} \int_{X_{N}}\left|\hat{f}_{1}\right| d \chi \text { by }(5) \\
& \leqslant B\left|X_{N}\right|^{(\theta-1) / 2+\beta+1 / 2}\left(\int_{X_{N}}\left|\hat{f}_{1}\right|^{2} d \chi\right)^{1 / 2}
\end{aligned}
$$

by Hölder's inequality,

$$
\begin{aligned}
& \leqslant B\left|X_{N}\right|^{\theta / 2+\beta}\left\|f_{1}\right\|_{2} \\
& \leqslant B\left|X_{N}\right|^{\theta / 2+\beta}\left|G_{N+1}\right|^{\theta / 2}\|f\|_{\infty}
\end{aligned}
$$

by the definition of $f_{1}$,

$$
\leqslant B \cdot b^{\theta / 2}\left|X_{N}\right|^{\beta}\|f\|_{\infty}
$$

Now clearly, 


$$
\begin{gathered}
\left.|| G_{N}\right|^{-1} \int_{G_{N}} k * f_{2} d x \mid \\
\leqslant\left.\left|G_{N}\right|^{-1} \int_{G_{N}}\left|k * f_{2}-\right| G_{N+1}\right|^{-1} \int_{G_{N+1}} k * f_{2} \mid d x \\
\quad+\left.|| G_{N+1}\right|^{-1} \int_{G_{N+1}} k * f_{2} \mid
\end{gathered}
$$

But

$$
\begin{array}{r}
\left.\left|G_{N}\right|^{-1} \int_{G_{N}}\left|k * f_{2}-\right| G_{N+1}\right|^{-1} \int_{G_{N+1}} k * f_{2} \mid d x \\
\leqslant\left|G_{N}\right|^{-1} \int_{G_{N}}\left|k * f_{2}-\sigma\right| d x+\left|G_{N+1}\right|^{-1} \int_{G_{N+1}}\left|k * f_{2}-\sigma\right| d x \\
\text { where } \sigma=\int_{G} k(-y) f_{2}(y) d y .
\end{array}
$$

The second term on the right of $(7)$ is equal to

$$
\left|G_{N+1}\right|^{-1} \int_{G_{N+1}} d x\left(\left|\int_{G \backslash G_{N+1}^{\theta}}(k(x-y)-k(-y)) f_{2}(y) d y\right|\right)=0
$$

since $x \in G_{N+1}$ and $k$ is constant on the cosets of $G_{N+1}$ outside $G_{N+1}^{\theta}$. The same argument shows that the first term on the right of (7) is also zero, so

$$
\left.\left.|| G_{N}\right|^{-1} \int_{G_{N}} k * f_{2} d x|\leqslant| G_{N+1}\right|^{-1} \int_{G_{N+1}} k * f_{2} d x \mid .
$$

Now write $f_{2}=f_{3}+f_{4}$ where $f_{3}=f_{2} \cdot \xi_{G_{N+2}^{e}}$. The argument used to estimate $\left|G_{N}\right|^{-1} \int_{G_{N}} k * f_{1} d x$ shows that

$$
\left.\left.|| G_{N+1}\right|^{-1} \int_{G_{N+1}} k * f_{3} d x\left|<B \cdot b^{\theta / 2}\right| X_{N+1}\right|^{\beta}\|f\|_{\infty}
$$

and the argument used to estimate $\left|G_{N}\right|^{-1} \int_{G_{N}} k * f_{2} d x$ shows that

$$
\left.|| G_{N+1}\right|^{-1} \int_{G_{N+1}} k * f_{4} d x|\leqslant|\left|G_{N+2}\right|^{-1} \int_{G_{N+2}} k * f_{4} d x \mid .
$$

We may suppose without loss of generality that $\hat{k}=0$ on $X_{M}$ for some large $M$; thus a continuation of the above argument leads to the estimate

$$
\begin{aligned}
\left.|| G_{N}\right|^{-1} \int_{G_{N}} k * f d x \mid & \leqslant b^{\theta / 2} \cdot B \cdot\|f\|_{\infty}\left(\sum_{N}^{M}\left|X_{n}\right|^{\beta}\right) \\
& \leqslant b^{\theta / 2} \cdot B \cdot\|f\|_{\infty} \sum_{0}^{\infty} 2^{-n \beta} \\
& \leqslant b^{\theta / 2} \cdot B \cdot\left(1-2^{-\beta}\right)^{-1}\|f\|_{\infty},
\end{aligned}
$$

which proves the lemma. 
Proof of Theorem. Set $\Theta(\chi)=\left|X_{n}\right|^{(\theta-1) / 2}$ when $\chi \in X_{n-1} \backslash X_{n}$, and consider the family of operators $U_{z}$ on $L^{2}(G)$ defined by

$$
\left(U_{z} f\right)^{\wedge}=\hat{f} \cdot \phi \cdot \Theta^{-z+\beta}
$$

where $0 \leqslant \operatorname{Re}^{\prime} z \leqslant 1$ and $\beta>0$. It is easy to check that, by virtue of (3), the mapping $z \rightarrow U_{z}$ is uniformly bounded and strongly continuous in the strip $0 \leqslant \operatorname{Re}^{\prime} z \leqslant 1$ and analytic in $0<\operatorname{Re}^{\prime} z<1$, to the space of bounded linear operators on $L^{2}(G)$.

It follows immediately from (3) that

$$
\left\|U_{1+i y} f\right\|_{2} \leqslant B \cdot\|f\|_{2} \text {. }
$$

Furthermore it follows from the above lemma that

$$
\left\|U_{i y} f\right\|_{\infty} \leqslant B \cdot C \cdot\|f\|_{\infty} .
$$

(This amounts essentially to the observation that a function constant on cosets of $X_{n}^{\theta}$ outside $X_{n}$ has Fourier transform constant on cosets of $G_{n}$ outside $G_{n}^{\theta}$.) An application of Stein's interpolation theorem, [6, p. 205], shows that

$$
\phi \cdot \Theta^{-t+\beta} \in M_{2 / t}(X)
$$

where $0<t<1$, and $\beta>0$. To see that $\phi \in M_{p}$ for all $p$ in $(1, \infty)$ fix $p$ in $(2, \infty)$ and set $t=2 / p$. Then

$$
\phi \Theta^{-2 / p+\beta} \in M_{p}(X),
$$

in particular when $\beta=2 / p$. The proof is complete.

Virtually the same proof yields:

COROLlary. Under the same conditions as stated in the theorem

$$
\phi \cdot \Theta^{-t} \in M_{p}(X) \text { when } 2 /(2-t)<p<2 / t \text {. }
$$

It is not difficult, using the ideas in [2], to construct examples of functions $\phi$ such that $\phi \cdot \Theta^{-t} \notin M_{p}$ when $p>2 / t$. The interesting case is, of course, when $p=2 / t$-see remark (b) below.

3. Remarks. (a) The condition that $\left|X_{n}\right| \cdot\left|X_{n+1}\right|^{-1} \leqslant b$ is not really necessary. If $b_{n}=\left|X_{n}\right| \cdot\left|X_{n+1}\right|^{-1}$ is such that $b_{n} \uparrow \infty$ and $\sum b_{n}^{\beta}<\infty$, then the above proof is easily adapted to show that:

If $\phi$ is a function constant on cosets of $X_{n}$ outside $X_{n}$ then $|\phi(\chi)| \leqslant B$. $b_{n}^{-(1 / 2+\beta)}$ when $\chi \in X_{n} \backslash X_{n+1}$ for some $\beta>0$ implies that $\phi \in A(X)$, and $|\phi(\chi)| \leqslant B \cdot b_{n}^{-1 / 2}$ when $\chi \in X_{n} \backslash X_{n+1}$ implies that $\phi \in M_{p}(X)$ for $1<p<$ $\infty$.

This complements some results of Spector [5]. Further results may be obtained by considering subsequences $\left\{X_{n_{k}}\right\}$ of $\left\{X_{n}\right\}$.

(b) There is a natural definition of $\operatorname{BMO}(G)$, the space of functions of bounded mean oscillation on $G$, see [2]. It would be interesting to know if the functions $\phi$ satisfying the hypotheses of the theorem are $L^{\infty} \rightarrow \mathrm{BMO}$ "multipliers". A positive answer would imply that $\phi \cdot \Theta^{-t} \in M_{2 / t}$ when $0<t<1$. 
(c) Let $Z$ denote the group of integers, and $Z_{n}$ the subgroup $2^{n} \cdot 2^{n-1}$ $\cdots \cdot 2^{1} Z$, where $n \geqslant 0$. Suppose $\phi$ is a function constant on cosets of $Z_{n+1}$ in $Z_{n} \backslash Z_{n+1}$. If $|\phi(m)| \leqslant B \cdot 2^{-(n+1)(\beta+1 / 2)}$ when $m \in Z_{n} \backslash Z_{n+1}$ for some $\beta>0$ then $\phi$ is a Fourier-Stieltjes transform, and if $|\phi(m)|<B \cdot 2^{-(n+1) / 2}$ when $m \in Z_{n} \backslash Z_{n+1}$ then $\phi \in M_{p}(Z)$ for $1<p<\infty$. To see this argue as follows: Let $X=\Delta_{a}$, the a-adic integers, where $a=(2,4,8,16, \ldots)$ (see [3, $\S 10])$, and let $X_{n}=\left\{x=\left(x_{j}\right)_{0}^{\infty} \in X: x_{j}=0\right.$ when $\left.0 \leqslant j \leqslant n-1\right\}$. It is easily seen that $\left|X_{n}\right|=\left(2^{n} \cdot 2^{n-1} \cdots \cdot 2^{1}\right)^{-1} \cdot X$ has an (algebraic!) subgroup isomorphic to $Z$, namely the group generated by the element $(1,0,0,0, \ldots)$, which we also denote by $Z$. Some rather tedious calculations show that the cosets of $Z_{n+1}$ in $Z_{n} \backslash Z_{n+1}$ sit inside the cosets of $X_{n}$ in $X_{n} \backslash X_{n+1}$; hence $\phi$ is the restriction of a function $\Phi$ on $X$, satisfying the hypotheses in (a) above. The result now follows immediately from well-known results about restrictions of Fourier multipliers to subgroups, see for example Saeki [4, Corollary 4.6].

\section{REFERENCES}

1. R. E. Edwards and G. I. Gaudry, Littlewood-Paley and multiplier theory, Springer-Verlag, Berlin and New York, 1977.

2. G. I. Gaudry and I. R. Inglis, Weak-strong convolution operators on certain disconnected groups, Studia Math. (to appear).

3. Edwin Hewitt and K. A. Ross, Abstract harmonic analysis, Vol. I, Springer-Verlag, Berlin, 1963. MR 28 \# 158.

4. Sadahiro Saeki, Translation invariant operators on groups, Tôhoku Math. J. 22 (1970), 409-419. MR 43 \#815.

5. René Spector, Sur la structure locale des groupes abéliens localement compacts, Bull. Soc. Math. France Suppl. Mém. 24 (1970). MR 44 \# 729.

6. E. M. Stein and Guido Weiss, Introduction to Fourier analysis on Euclidean spaces, Princeton Math. Ser., no. 32, Princeton Univ. Press, Princeton, N.J., 1971. MR 46 \#4102.

Istituto Matematico, Università di Milano, 20133 Milano, Italy 